\title{
Evidence of Bordetella pertussis infection in vaccinated 1-year-old Danish children
}

\author{
Marie-Louise von Linstow • Peter Lotko Pontoppidan • \\ Carl-Heinz Wirsing von König • James D. Cherry • \\ Birthe Hogh
}

Received: 30 December 2009/Accepted: 16 March 2010/Published online: 8 April 2010

(C) The Author(s) 2010. This article is published with open access at Springerlink.com

\begin{abstract}
We measured IgA and IgG antibodies to pertussis toxin (PT) and filamentous hemagglutinin (FHA) in sera from 203 1-year-old children who had received one to three doses of a monocomponent PT toxoid vaccine. Ten children (5\%) had IgA antibody to PT indicating recent infection; seven of these children had received three doses of vaccine. PT IgA responders did not have significantly longer coughing episodes than PT IgA non-responders. Since an IgA antibody response occurs in only $\sim 50 \%$ of infected children, the actual infection rate in our cohort is estimated to $\sim 10 \%$. The apparent high Bordetella pertussis infection rate in Danish infants suggests that the monocomponent PT toxoid vaccine used in Denmark has limited efficacy against $B$. pertussis infection. A prospective immunization study comparing a multi-component vaccine with the present monocomponent PT toxoid vaccine should be undertaken.
\end{abstract}

Keywords Pertussis · Pertussis toxin · Pertussis IgA · Pertussis epidemiology

\footnotetext{
M.-L. von Linstow · P. L. Pontoppidan · B. Hogh

Department of Pediatrics, Hvidovre Hospital,

University of Copenhagen,

Copenhagen, Denmark

C.-H. W. von König

Institut für Hygiene und Laboratoriumsmedizin,

Krefeld, Germany

\section{J. D. Cherry $(\bowtie)$}

Division of Infectious Diseases,

Mattel Children's Hospital UCLA,

Los Angeles, CA, USA

e-mail: jcherry@mednet.ucla.edu
}

\section{Introduction}

Despite high vaccination coverage, pertussis continues to be a major cause of morbidity and mortality in Europe [16]. The clinical spectrum is diverse and is affected by patient age, previous exposure to the organism and immunization history $[2,3,11]$. The heterogeneity in pertussis disease expression makes it an often unrecognized and undiagnosed disease. Pertussis appears epidemically every 2 to 5 years. However, epidemiological data are incomplete, and different reporting procedures suggest that only the tip of the iceberg is evident $[2,3,11,16]$. Improved disease surveillance and diagnoses are essential to determine the true burden of the disease.

The situation in Denmark is unique in that all children are vaccinated with a domestically produced DTaP (Di-TeKik) vaccine in which toxoided pertussis toxin (PT) is the only pertussis component. The PT toxoid vaccine replaced the whole-cell pertussis vaccine in January 1997. The PT toxoid vaccine is reported to provide about $78 \%$ protection against notified pertussis after three vaccinations given at 3,5 and 12 months of age as part of the national child immunization program [9]. Vaccination effectiveness for two doses of pertussis toxoid vaccine in the first year of life is reported to be $59 \%$. In September 2003, a pre-school pertussis booster vaccination at 5 years of age was implemented to reduce transmission to susceptible young children. Infection results in IgA and IgG antibody responses to specific Bordetella pertussis antigens, whereas vaccination results in only an $\operatorname{IgG}$ response to these antigens $[3,11]$.

Diagnosis of pertussis based on clinical symptoms is complicated by a number of factors: the wide spectrum of 
symptoms and misdiagnosis owing to similarity between symptoms of other infections $[2,3,11,16]$. A WHO committee in 1991 developed a primary case definition to be used in vaccine efficacy trials: An illness with paroxysmal cough of $\geq 21$ days and either culture-confirmed infection with $B$. pertussis or serological evidence of infection with $B$. pertussis or household contact with a case of cultureconfirmed pertussis with onset of cough within 28 days before or after onset of cough [21].

In the present study, we investigated whether there was evidence of circulation of $B$. pertussis in the community. This would provide baseline data on which to assess the epidemiology of B. pertussis and thus direct future use of pertussis vaccines in Denmark.

\section{Methods}

Study subjects The study population consisted of healthy children enrolled from the postnatal ward at Hvidovre Hospital, Denmark during a 12-month period from May 2004 to May 2005 [19]. Children were originally enrolled for the study of human metapneumovirus and respiratory syncytial virus infections in infancy. Approximately 20 children were enrolled each month to ensure that the children were sampled equally throughout the year. A total of 242 healthy newborns were enrolled, of whom 217 were followed throughout 1 year.

Study procedures Children were monitored with detailed health diaries and through monthly home visits until 1 year of age. The study procedures have been previously described in detail [19]. The children were scheduled to receive routine immunizations with D-T-aP-IPV-Hib at 3, 5 and 12 months of age. Blood samples were obtained by venous puncture from 203 of the children at age 12 months and the sera kept at $-80^{\circ} \mathrm{C}$ until further analysis.

Laboratory assays Twofold dilutions of $50 \mu \mathrm{l}$ of serum were used for the pertussis ELISA. IgA and IgG antibodies to PT and filamentous hemagglutinin (FHA) were measured by a standardized ELISA procedure and expressed in ELISA units $(\mathrm{EU} / \mathrm{ml})$ with respect to reference serum 3 of the Center for Biologics Evaluation and Research/US Food and Drug Administration (CBER/FDA) [20]. The lower levels of detection for the assays were $1.7 \mathrm{EU} / \mathrm{ml}$ for IgGanti-PT, 2.4 EU/ml for IgA-anti PT, $1.8 \mathrm{EU} / \mathrm{ml}$ for IgG-antiFHA and $1.7 \mathrm{EU} / \mathrm{ml}$ for IgA-anti-FHA.

Statistical analysis Antibody concentrations and prevalence were tabulated. For differences in duration in days of coughing episodes and in infants with IgA positive and IgA negative samples, the Mann-Whitney rank sum was used.

\section{Results}

At the time of blood sampling, $100 \%$ of children had received one dose, $98 \%$ had received two doses and $23 \%$ had received three doses of the PT toxoid vaccine. Because IgA antibodies are a result of infection and not vaccination, we used their presence as an indicator of infection in the study children. Ten children (4.9\%) had IgA antibody to PT, 18 (8.9\%) had IgG antibody to FHA, 193 (95.1\%) had IgG antibody to PT and 13 (6.4\%) had IgA antibody to FHA. Seven of the ten PT IgA responders had received three doses of vaccine and the remaining three PT $\operatorname{IgA}$ responders had received two doses at 3 and 5 months of age. The median PT IgG titer for fully vaccinated children was $50 \mathrm{EU} / \mathrm{ml}$ vs. $8 \mathrm{EU} / \mathrm{ml}$ for children who had only received one or two doses of the PT toxoid vaccine $(p<0.001)$.

Clinical data on cough were obtained from the health diaries of the study subjects and are illustrated in Table 1. PT IgA responders did not have significantly longer coughing episodes during the first year of life than $\operatorname{IgA}$ non-responders (median 11.5 days vs. 10.0 days, $p=0.186$ ). However, two (20\%) of IgA responders had cough for more than 21 days compared to 27 (14\%) of IgA non-responders $(p=>0.05)$. Mean age at longest coughing episode was 7.5 months. In one PT IgA positive and 20 PT IgA negative children, the longest coughing episode was associated with wheezing, suggesting a viral infection and not $B$. pertussis infection as the cause of the illnesses. Excluding these 21 children from analysis did not alter the main results (median duration of cough 11 vs. 9 days, $p=0.209$ ). No differences in other symptoms such as fever, rash, diarrhea and vomiting were observed between the groups (data not shown).

\section{Discussion}

Infection with $B$. pertussis stimulates both $\operatorname{IgA}$ and $\operatorname{IgG}$ antibody responses to $B$. pertussis antigens. In an efficacy

Table 1 Duration of longest coughing episode during the first year of life in 203 Danish children related to PT IgA antibody status

\begin{tabular}{llc}
\hline Duration of cough & $\begin{array}{l}\text { PT IgA pos } \\
(n=10)(\%)\end{array}$ & $\begin{array}{l}\text { PT IgA neg } \\
(n=193)(\%)\end{array}$ \\
\hline$<7$ days & $2(20)$ & $59(30.6)$ \\
$7-14$ days & $4(40)$ & $89(46.1)$ \\
$15-21$ days & $2(20)$ & $18(9.3)$ \\
$22-28$ days & $1(10)$ & $8(4.1)$ \\
$29-35$ days & 0 & $11(5.7)$ \\
$36-42$ days & 0 & $3(1.6)$ \\
$43-49$ days & $1(10)$ & $2(1.0)$ \\
$>49$ days & 0 & $3(1.6)$ \\
\hline
\end{tabular}


trial [12] in Germany, only 120 (52\%) of 232 children with pertussis had an IgA response to PT (Heininger and Cherry unpublished data 1998). We found serological evidence of B. pertussis infection (IgA PT antibody) in 5\% of our cohort of vaccinated healthy children during their first year of life. Since only $50 \%$ of infections result in a PT $\operatorname{IgA}$ antibody response, the actual infection rate in our cohort may have been $\sim 10 \%$. It is of interest that seven of 47 $(15 \%)$ with three doses of vaccine had a PT IgA antibody response, whereas only three of the $156(2 \%)$ who had less than three doses of vaccine had a PT IgA antibody response. The explanation for this observation is that once a natural infection has occurred, immunization will enhance the PT IgA response. This was observed following the fourth dose in the German efficacy trial mentioned above [12] as well as in a more recent adult vaccine efficacy trial [10]. The presence of IgG and IgA antibodies to FHA in $8.9 \%$ and $6.4 \%$, respectively, in the serum of study subjects is also an indicator of $B$. pertussis infection since the vaccine did not contain this antigen. However, antibody to FHA in contrast to antibody to PT is less specific. Infection with other Bordetella sp. as well as other gram negative bacteria may stimulate an antibody response to FHA [18].

Illnesses with cough are common during infancy. The duration of the most significant cough illness in our study subjects with evidence of $B$. pertussis infection was not significantly different from the duration in subjects without evidence of $B$. pertussis infection. This observation suggests that the infections that occurred in the previous vaccinees were clinically mild.

With the exception of the DTaP vaccine (Di-Te-Kik) in use in Denmark, which contains only toxoided PT as the pertussis component, the $\mathrm{DTaP}$ vaccines presently in use throughout the world contain toxoided PT as well as $1-4$ other B. pertussis antigens (FHA, pertactin [PRN] and fimbriae $2 / 3$ [FIM 2/3]) [3, 11]. The reported pertussis vaccine efficacy in the 1980s and 1990s trials varied considerably based on the case definition used [3-7, 11-15, 17].

The main objection to the WHO primary case definition of pertussis is that it eliminated many laboratory-confirmed clinically mild cases from efficacy calculations in vaccine trials. Because mild cases are more common in vaccinees than in controls, efficacy is inflated. Therefore, vaccine efficacy appears better than it truly is, and less effective vaccines would also seem comparable with their more effective counterparts. Results from a randomized trial of acelluar pertussis vaccines in Stockholm in the 1980s showed that significantly fewer recipients of a twocomponent vaccine (JNIH-6) were diagnosed as primary or co-primary cases in households than those who received a monocomponent vaccine (JNIH-7) [13]. Regarding the Danish PT toxoid vaccine (Di-Te-Kik), it was used exclusively in the Gothenburg region of Sweden from
1996 until the spring of 1999. The rate of pertussis (in children born between January 1996 and June 1999) between October 1997 and September 2004 was determined for children in the Gothenburg region and the rest of Sweden [1, 8]. The rate of pertussis (mostly vaccine failures) in the Gothenburg region was significantly higher in all children less than 8 years than the rate in the rest of Sweden, where a three component DTaP vaccine was predominantly used.

In conclusion, our data indicate that $5-10 \%$ of our vaccinated cohort of healthy children had a $B$. pertussis infection during the first year of life, demonstrating that $B$. pertussis is circulating in the community even though few clinical cases were recognized. The high $B$. pertussis infection rate in Danish infants as well as the surveillance data from Sweden suggest that the PT toxoid vaccine used in Denmark has less than optimal efficacy. A prospective immunization study comparing a multi-component vaccine with the present monocomponent PT toxoid vaccine should be undertaken.

Conflict of interest The laboratory studies in this paper were supported by an unrestricted grant from Sanofi pasteur. Carl-Heinz Wirsing von König and James D. Cherry have been consultants to Sanofi pasteur and have been participants in the Global Pertussis Initiative which is supported by Sanofi pasteur. Dr. Cherry is a member of the Sanofi pasteur speaker's bureau.

Open Access This article is distributed under the terms of the Creative Commons Attribution Noncommercial License which permits any noncommercial use, distribution, and reproduction in any medium, provided the original author(s) and source are credited.

\section{References}

1. Carlsson RM, Gustafsson L (2008) Ten year report. Pertussis surveillance in Sweden. Progress Report 1 October 1997-31 December 2007. Smittskyddsinstitutets rapportserie 2008; No. 4

2. Cherry JD, Grimprel E, Guiso N, Heininger U, Mertsola J (2005) Defining pertussis epidemiology clinical, microbiologic and serologic perspectives. Pediatr Infect Dis J 24:S25-S34

3. Cherry JD, Heininger U (2009) Pertussis and other Bordetella infections. In: Feigin RD, Cherry JD, Demmler-Harrison GJ, Kaplan S (eds) Feigin \& Cherry's textbook of pediatric infectious diseases, 6th edn. WB Saunders Co, Philadelphia, pp 1683-1706

4. Cherry JD, Olin P (1999) The science and fiction of pertussis vaccines. Pediatrics 104:1381-1383

5. Cherry JD (1997) Comparative efficacy of acellular pertussis vaccines: an analysis of recent trials. Pediatr Infect Dis J 16:S90-S96

6. Greco D, Salmaso S, Mastrantonio P et al (1996) A controlled trial of two acellular vaccines and one whole-cell vaccine against pertussis. N Engl J Med 334:341-348

7. Gustafsson L, Hallander HO, Olin P, Reizenstein E, Storsaeter J (1996) A controlled trial of a two-component acellular, a fivecomponent acellular, and a whole-cell pertussis vaccine. $\mathrm{N}$ Engl $\mathrm{J}$ Med 334:349-355 
8. Hallander HO, Gustafsson L (2009) Efficacy and effectiveness of acellular pertussis vaccines: a 20-year Swedish experience. Expert Rev Vaccines 8:1303-1307

9. Hviid A, Stellfeld M, Andersen PH, Wohlfahrt J, Melbye M (2004) Impact of routine vaccination with a pertussis toxoid vaccine in Denmark. Vaccine 22:3530-3534

10. Le T, Cherry JD, Chang S-J et al (2004) Immune responses and antibody decay after immunization of adolescents and adults with an acelluar pertussis vaccine: The APERT Study. JID 190:535-544

11. Mattoo S, Cherry JD (2005) Molecular pathogenesis, epidemiology, and clinical manifestations of respiratory infections due to Bordetella pertussis and other bordetella subspecies. Clin Microbio Rev $18: 326-382$

12. Stehr K, Cherry JD, Heininger U et al (1998) A comparative efficacy trial in Germany in infants who received either the Lederle/Takeda acelluar pertussis component DTP (DTaP) vaccine, the Lederle whole-cell component DTP vaccine, or DT vaccine. Pediatrics 101:1-11

13. Storsaeter J, Blackwelder WC, Hallander HO (1992) Pertussis antibodies, protection, and vaccine efficacy after household exposure. Am J Dis Child 146:167-172

14. Storsaeter J, Hallander H, Farrington CP, Olin P, Möllby R, Miller E (1990) Secondary analyses of the efficacy of two acellular pertussis vaccines evaluated in a Swedish phase III trial. Vaccine 8:457-461
15. Storsaeter J, Olin P (1992) Relative efficacy of two acellular pertussis vaccines during three years of passive surveillance. Vaccine 10:142-144

16. Tan T, Trindade E, Skowronski D (2005) Epidemiology of pertussis. Pediatr Infect Dis J 24:S10-S18

17. Trollfors B, Taranger J, Lagergard T et al (1995) A placebocontrolled trial of a pertussis-toxoid vaccine. N Engl J Med 333:1045-1050

18. Vincent JM, Cherry JD, Nauschuetz WF et al (2000) Prolonged afebrile nonproductive cough illnesses in American Soldiers in Korea: a serological search for causation. Clin Infect Dis 30:534539

19. von Linstow ML, Hogh M, Nordbo SA, Eugen-Olsen J, Koch A, Hogh B (2008) A community study of clinical traits and risk factors for human metapneumovirus and respiratory syncytial virus infection during the first year of life. Eur $\mathrm{J}$ Pediatr 167:1125-1133

20. Wirsing von Konig CH, Gounis D, Laukamp S, Bogaerts H, Schmitt HJ (1999) Evaluation of a single-sample serological technique for diagnosing pertussis in unvaccinated children. Eur J Clin Microbiol Infect Dis 18:341-345

21. World Health Organization (1991) World Health Organization Meeting on Case Definition of Pertussis, Geneva, January 10-11, 1991. MIM/EPI/PERT/9.1 Geneva, World Health Organization 